\title{
Comparative Study of UWB Communications over Fiber using Direct and External Modulations
}

\author{
V. Khanaa ${ }^{1 *}$, Krishna Mohanta ${ }^{2}$ and T. Saravanan ${ }^{3}$ \\ 1,2Department of CSE, Sri Sai Ram Engg. College, Leo Nagar, Chennai-600044, India; \\ drvkannan62@yahoo.com \\ 1,2Department of ECE, Bharath University, Chennai-600073, India; krishnamohanta@gmail.com; \\ ${ }_{3}^{3}$ Professor \& Head, Department of ETC, Bharath University, Chennai-600073, India; saravanan.etc@bharathuniv.ac.in
}

\begin{abstract}
This work presents an analysis of the Ultra Wide Band (UWB) Radio Frequency transmission over fiber using two types of modulation, direct and external. Both the modulation schemes are simulated by using OPTSIM 5.3 and optimized.
\end{abstract}

Keywords: UWB, MB-OFDM, Radio Over Fiber, MZM, VCSEL, Q-Factor.

\section{Introduction}

Today's increasingly connected world has a continuous need to share information and resources. The demand for high-capacity multimedia services in real- time needs wireless broadband access. In order to meet this requirement, a fiber based wireless access using Radio-over-Fiber (RoF) technology is necessary. The evolution of high-speed, highbandwidth telecommunication technologies has resulted in entirely new approaches to work, study, and research that rely on various levels of networking architectures to deliver appropriate connectivity to the end-user. The advent of these high-speed, high-bandwidth networks has also driven a new fundamental need: real-time communication and interaction.

Ultra Wide Band (UWB) radio communications have attracted growing attention due to its promising capabilities to provide high data rate with low cost and low power consumption [1-2]. Low transmitted power of UWB signals limits the wireless range of these systems, and one way to extend the range is to use radio over fiber (RoF) systems as a distributed antenna structure. In RoF systems, analog radio signals are modulated on the intensity of the optical signals and transmitted through a fiber optic link.
The Ultra Wide Band Radio over Fiber (UWB RoF) is a novel technology for the transmission of UWB signals by using light as carrier propagating through an optical fiber. In this approach, the UWB RF signal itself is superimposed on the optical CW carrier. This strategy makes the conversion process transparent to the UWB's modulation method, and also transparency feature allows avoiding the high costs of additional electronic components required for synchronization and other processes. The development of RoF systems is motivated by the demand for replacing a central high power antenna with a low power Distributed Antennas System (DAS). RoF systems are usually composed of many Base Stations (BSs) which are connected to a single Central Station (CS) [3-7].

Transmission of UWB signals through optical fiber have been a topic of interest for many researchers for the past few years, however most of the studies have focused on the feasibility and the experimental demonstration of such systems. Here UWB over fiber link is measured to investigate the transmission of MB-OFDM signals. In this paper, a comparison between both modulation techniques, i.e., external modulation with MZM and the direct modulation of VCSEL laser are carried out.

\footnotetext{
* Corresponding author:

V. Khanaa (drvkannan62@yahoo.com)
} 


\section{Simulation Setup and Description}

A RoF Optical Communication System using direct modulation method as well as external intensity modulation has been set up using OPTSIM version 5.3. The simulation setup is shown in the Figure 1 and Figure 2.

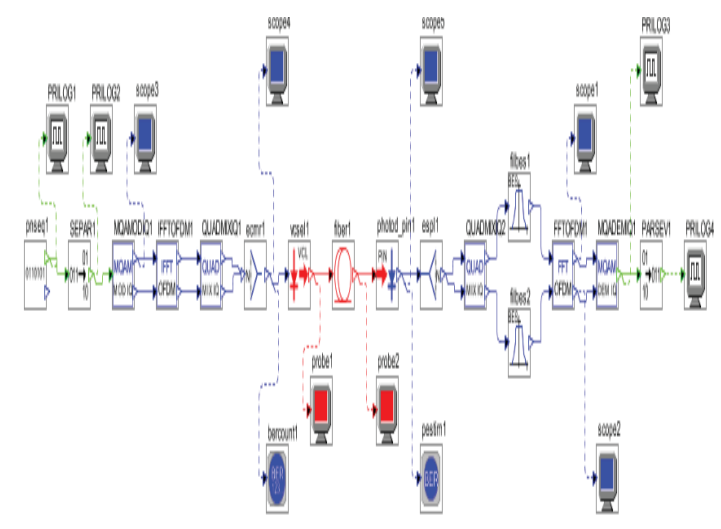

Figure 1. Simulation Setup 1.

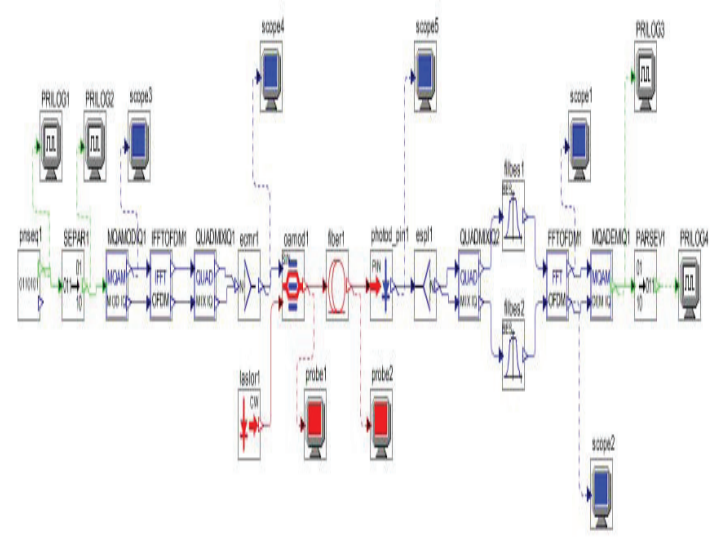

Figure 2. Simulation Setup 2.

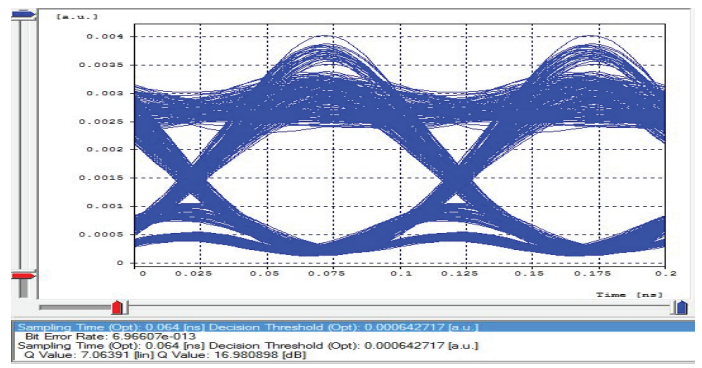

Figure 3. Eye Diagrams of the (Direct Modulation) system before optical transmission and after optical reception for $10 \mathrm{Gbps}$ input data.

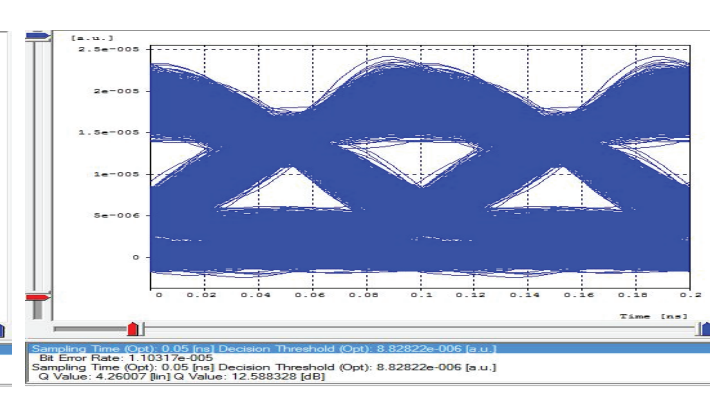

In a direct modulation, the RF signals transmitting from the transmitter is fed to a direct modulated laser (here VCSEL) where it is directly modulated with laser's drive current. A 10 Gbps data source is used. The modulated signal is transmitted through optical fiber of $10 \mathrm{~km}$ length. At the receiving section a PIN photodiode is employed in order to convert optical signal into electrical form. The electrical signals are fed to the OFDM receiver to recover the data and the output data is observed through a logical measurement scope [4-9].

In external modulation, the RF signals transmitting from the transmitter and the optical signal from a CW Laser are fed to an external Modulator (here Mach Zehnder Modulator). A $10 \mathrm{Gbps}$ data source is used. The modulated signal is transmitted through optical fiber of $10 \mathrm{~km}$ length. At the receiving section a PIN photodiode is employed in order to convert optical signal into electrical form. The electrical signals are fed to the OFDM receiver to recover the data. The output data is observed through a logical measurement scope.

\section{Results and Discussion}

The performance of direct intensity modulation and external modulation has investigated in terms of Bit error rate, $\mathrm{Q}$ value and eye diagrams. The Figure 3 and Figure 4 shows the eye diagrams of optical communication system by using direct modulation and external intensity modulation respectively. The Figure 5 shows the Q estimation chart of the system with respect to bit rate by using direct modulation and external intensity modulation respectively. The Figure 6 shows the BER chart with respect to bit rate by using direct modulation and external intensity modulation respectively. The Figure 7 and Figure 8 shows the logical output diagrams at 10 Gbps input by using direct modulation and external intensity modulation respectively. Comparing the characteristics of the system by using direct modulation and external 

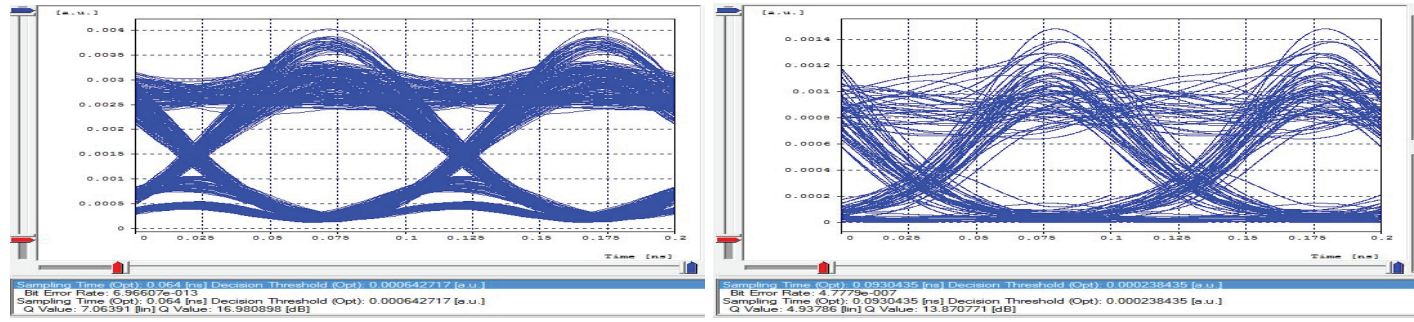

Figure 4. Eye Diagrams of the (External Modulation) system before optical transmission and after optical reception for 10 Gbps input data.

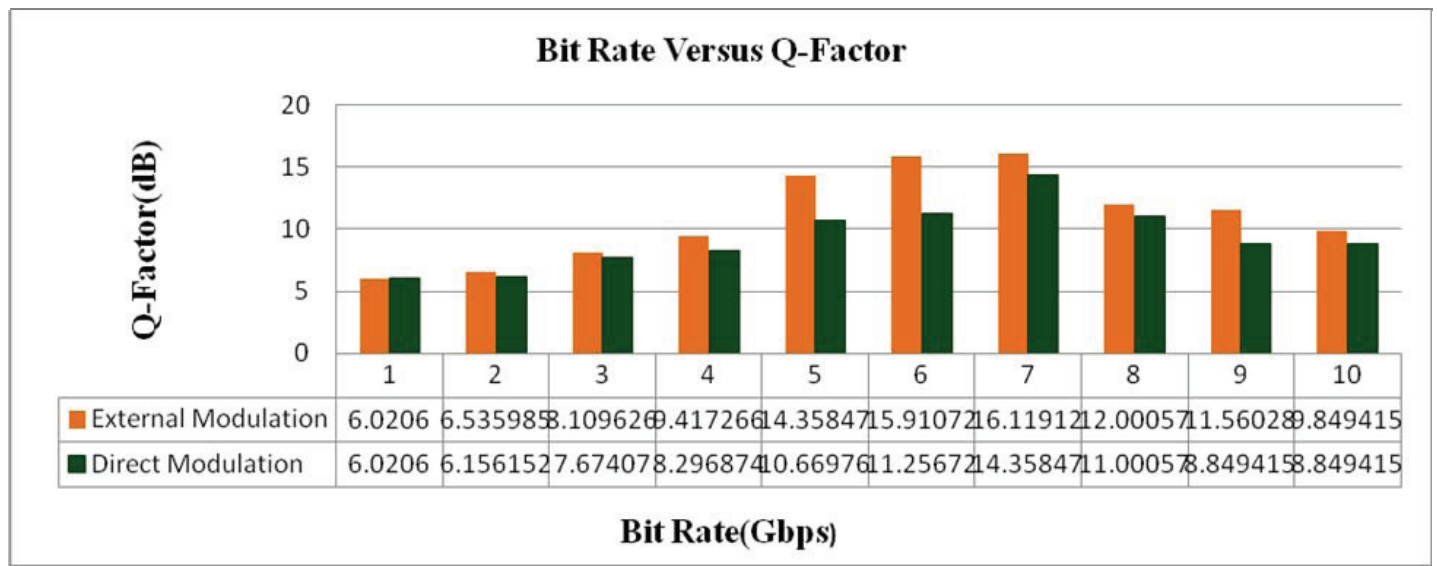

Figure 5. Q value estimation chart.

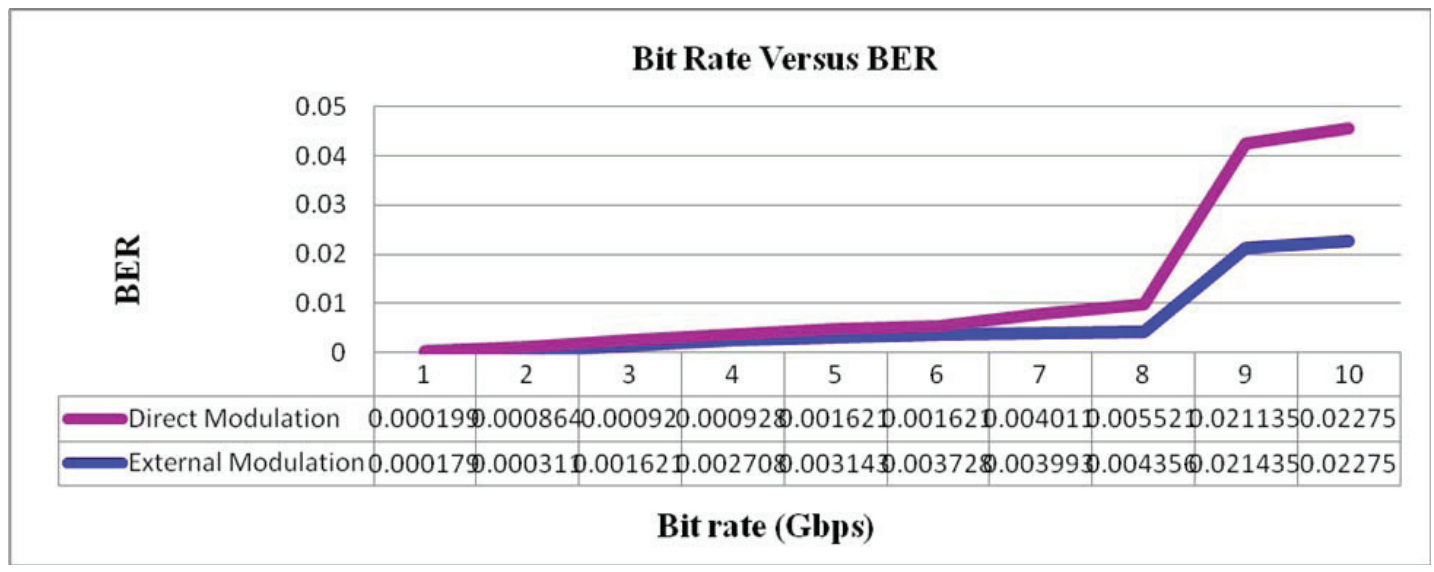

Figure 6. BER chart.

intensity modulation it can be seen that external intensity modulation have better Q value and BER [10-15].

\section{Conclusion}

The UWB technology offers many advantages over narrowband techniques. High-speed connections, interference protection and simple hardware architectures are a few of the characteristics that are propelling the rapid growth of UWB devices. In this work the link performances of UWB RoF using two types of modulation, direct and external was investigated. The result obtained from OptSim simulation confirms the efficiency of external modulation techniques over the direct modulation techniques. 


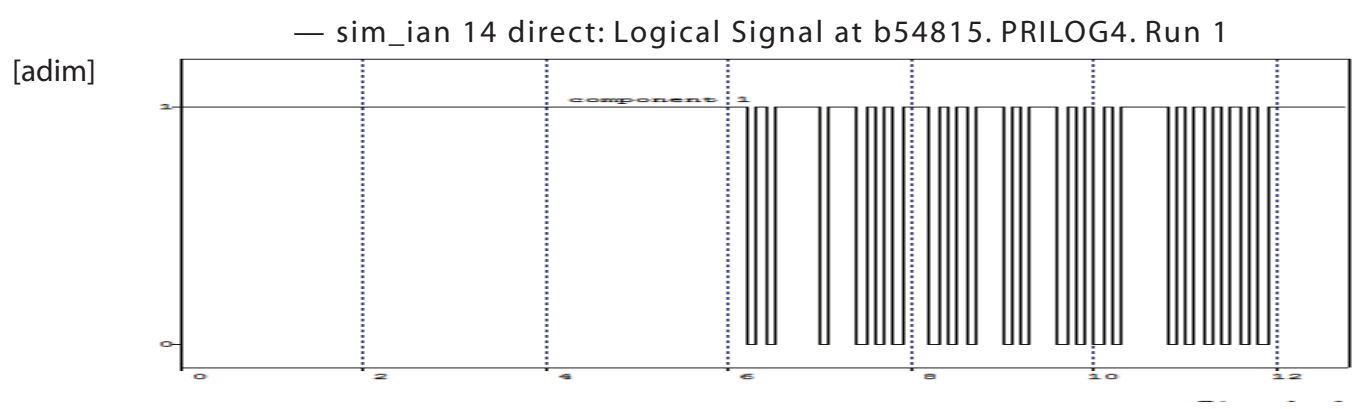

Figure 7. Logical output data (Direct Modulation) for 10 Gbps input data.

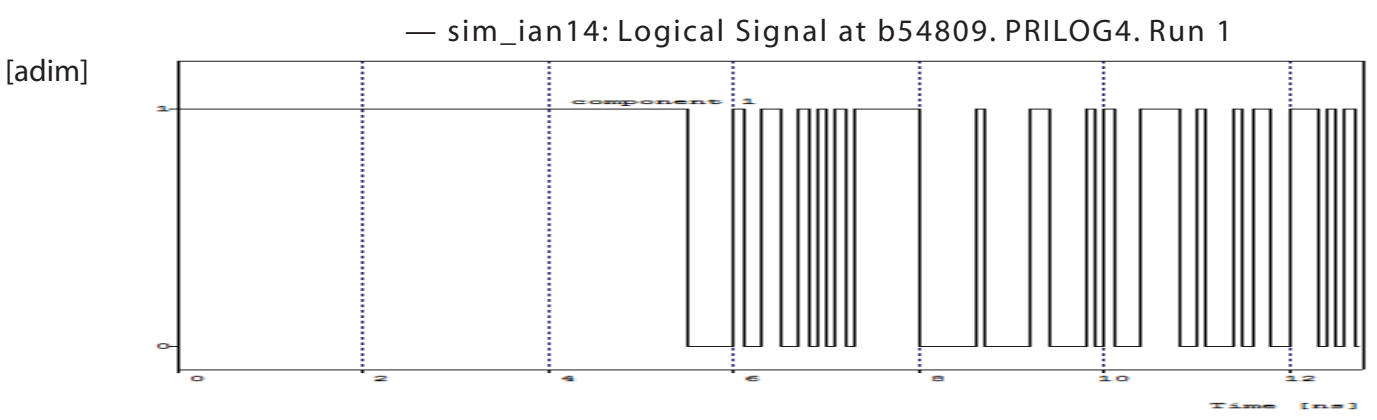

Figure 8. Logical output data (External Modulation) for 10 Gbps input data.

\section{References}

1. Billabert A, Deshours F et al. (2009). Modulator non linearity influence on UWB signal performance over RoF link, Proceedings of the 6th European Microwave Integrated Circuits Conference, vol 1, 8-43

2. Batra A, Balakrishnan J et al. (2004). Design of a multiband OFDM system for realistic UWB channel environments, IEEE Transactions on Microwave Theory and Techniques, vol 52, No. 9, 2123-2138

3. Cox C III, Ackerman E et al. (1997). Techniques and performance of intensity-modulation direct-detection analog optical links, IEEE Transactions on Microwave Theory and Techniques, vol 45, No. 8, 1375-1383.

4. Wentzloff D D, Blázquez R et al. (2005). System design considerations for ultra-wideband communication, IEEE Communications Magazine, vol 43(8), 114-121.

5. Wilson J M (2002). Ultra-wideband a disruptive RF technology?, Intel Research \& Development, 3-8.

6. Andrews J R (2003). UWB signal sources, antennas \& propagation, IEEE Topical Conference on Wireless Communication Technology, 439-440.

7. Gerrits J F M, Kouwenhoven M H L et al. (2005). Principles and limitations of ultra-wideband FM communications systems, EURASIP Journal on Applied Signal Processing, vol 3, 382-396.

8. Kalpna K, and Jain B (2011). Performance evaluation \& optimization of error free WDM radio over fiber link,
International Journal of Engineering Science and Technology, vol 3(5), 4051-4060.

9. Omomukuyo O, and Mitchell J E (2012). Performance evaluation of a 60-ghz multi-band OFDM (MB-OFDM) ultra-wideband radio-over-fiber system, University College London.

10. Horváth P, and Frigyes I (2005). Effects of the nonlinearity of a Mach-Zehnder modulator on OFDM radio-over-fiber transmission, IEEE Communications Letters, vol 9, No. 10, 921-923.

11. Malhotra R, and Tanvi (2009) UWB communication receiver: review and design considerations, International Journal of Advanced Engineering Sciences and Technologies, vol 8(2), 197-202.

12. Kaur S, and Bharti G (2012). Orthogonal frequency division multiplexing in wireless communication systems: A Review, International Journal of Advanced Research in Computer Engineering \& Technology, vol 1(3).

13. Chan S (2008). Frequency division multiplexed radio-overfiber transmission using an optically injected laser diode, Proceedings of SPIE, vol 6997, 69971Y-1-69971Y-9.

14. Cseh T, Berceli T et al. (2012). Optical transmission of OFDM m-QAM RF signals: comparison of direct and external modulations, 2012 14th International Conference on Transparent Optical Networks (ICTON), 1-5.

15. Zhang Y (2011). Development of millimeter-wave radioover-fiber technology, Journal Of Electronic Science And Technology, vol 9, No. 1, 58-66. 